\title{
Identification of a Hyperparasitic Simplicillium obclavatum Strain Affecting the Infection Dynamics of Puccinia striiformis f. sp. tritici on Wheat
}

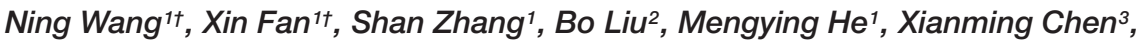 \\ Chunlei Tang ${ }^{1}$, Zhensheng Kang ${ }^{1}$ and Xiaojie Wang ${ }^{1 *}$ \\ ' State Key Laboratory of Crop Stress Biology for Arid Areas and College of Plant Protection, Northwest A\&F University, \\ Shaanxi, China, ${ }^{2}$ State Key Laboratory for Biology of Plant Disease and Insect Pests, Institute of Plant Protection, Chinese \\ Academy of Agricultural Sciences, Beijing, China, ${ }^{3}$ USDA-ARS, Wheat Genetics, Physiology, Quality, and Disease Research \\ Unit and Department of Plant Pathology, Washington State University, Pullman, WA, United States
}

OPEN ACCESS

Edited by:

Rajesh Jeewon,

University of Mauritius, Mauritius

Reviewed by:

George Newcombe,

University of Idaho, United States Birinchi Kumar Sarma

Banaras Hindu University, India

${ }^{*}$ Correspondence: Xiaojie Wang

wangxiaojie@nwsuaf.edu.cn

${ }^{\dagger}$ These authors have contributed equally to this work

Specialty section: This article was submitted to Fungi and Their Interactions,

a section of the journal Frontiers in Microbiology

Received: 15 February 2020 Accepted: 19 May 2020

Published: 24 June 2020

Citation:

Wang N, Fan X, Zhang S, Liu B, He M, Chen X, Tang C, Kang $Z$ and Wang $X$ (2020) Identification of a Hyperparasitic Simplicillium

obclavatum Strain Affecting the Infection Dynamics of Puccinia striiformis $f$. sp. tritici on Wheat. Front. Microbiol. 11:1277. doi: 10.3389/fmicb.2020.01277
Wheat stripe rust, caused by Puccinia striiformis f. sp. tritici (Pst), is one of the most serious threats to wheat production worldwide. Changes of Pst virulence may circumvent resistance in wheat varieties, and application of fungicides may cause environmental problems. Parasites of Pst can be used to develop biological agents for environmentally friendly control of this fungal disease. Here, we report a hyperparasitic fungus isolated from Pst and identified it as Simplicillium obclavatum through molecular and morphological characterizations. We demonstrated that inoculation of Pst-infected wheat leaves with S. obclavatum reduced the production and germination rate of Pst urediniospores. Therefore, S. obclavatum has the potential to be developed into a biological control agent for managing wheat stripe rust.

Keywords: wheat stripe rust, Puccinia striiformis, Simplicillium obclavatum, hyperparasite, biological control

\section{INTRODUCTION}

Cereal rust outbreaks have caused significantly economic losses throughout the history of grain production. Almost all important grain crops, including wheat, rye, barley, oat, and other cereal crops, can be infected by rust fungi (Saari and Prescott, 1985). Furthermore, global grain production and worldwide food security are still severely challenged by cereal rust diseases (Fisher et al., 2012). Thus, development of tactics for control of cereal rust diseases is essential for food security. Biological control is one of the most environmentally friendly approaches for controlling plant pathogens but has not been intensively explored for managing cereal rusts.

Many biological antagonists can be effective against rust fungi (Bettiol and Várzea, 1992; Shiomi et al., 2006; Haddad et al., 2009; Jackson et al., 2012; Zheng et al., 2017). Fungi in several genera are hyperparasitic on rust fungi (Littlefield, 1981; Zheng et al., 2017). In the genus of Cladosporium, C. uredinicola, C. cladosporioides, C. pseudocladosporioides, C. aecidiicola, and C. sphaerospermum have been reported to parasitize rust fungi of the order Pucciniales (Keener, 1954; Srivastava et al., 1985; Vandermeer et al., 2009; Torres et al., 2017; Sun et al., 2019). Fungal species C. cladosporioides, Eudarluca caricis, Microdochium nivale, Typhula idahoensis, 
Lecanicillium lecanii, and Alternaria alternata have been reported to infect urediniospores of Puccinia (Littlefield, 1981; Nischwitz et al., 2005; Zhan et al., 2014; Zheng et al., 2017). For instance, Lecanicillium lecanii can parasitize the coffee rust pathogen, Hemileia vastatrix, reducing rust severity (Vandermeer et al., 2009; Jackson et al., 2012; Martins et al., 2015). Simplicillium lanosoniveum has been reported either as an entomopathogen or as a mycoparasite recovered from scale insects or rust fungal spores on coffee plants (Zare and Gams, 2001). As a biological control agent for soybean rust pathogen Phakopsora pachyrhizi, S. lanosoniveum was found to reduce the development of new uredinia and the germination rate of urediniospores (Ward et al., 2012). Baiswar et al. (2014) found that S. lanosoniveum also parasitizes rust pustules on Elaeagnus latifolia. Thus, biocontrol strategies have the potential for control of rusts.

One of the most important and destructive diseases on wheat is stripe rust, also called yellow rust, caused by Puccinia striiformis f. sp. tritici (Pst) (Chen, 2005; Wan et al., 2007). Yellow rust is named after yellow uredinia formed on leaf surfaces and other above ground parts of wheat. The previous studies reported that $M$. nivale, L. lecanii, T. idahoensis, C. cladosporioides, and A. alternata could parasitize Pst uredinia and urediniospores (Littlefield, 1981; Zhan et al., 2014; Zheng et al., 2017). We found that Pst uredinia were surrounded by white fungal hyphae during Pst urediniospore production, and white hyphae almost engulfed all Pst uredinia. Eventually, uredinia completely disappeared. Previous studies showed that hyperparasites stopped Pst sporulation (Zhan et al., 2014; Zheng et al., 2017).

Identification of parasites infecting cereal pathogenic fungi is essential for developing biological control strategies for managing plant diseases. In the present study, we report the discovery of a fungal strain isolated from Pst. We demonstrated that the fungus was able to parasitize the obligate biotrophic rust fungus. Through molecular and morphological characterizations, we identified the hyperparasitic fungus as species Simplicillium obclavatum. Our experiments showed that S. obclavatum was able to impair Pst sporulation and also reduce urediniospore germination. Therefore, the isolate of $S$. obclavatum has the potential to be developed as a biological control agent for managing stripe rust.

\section{MATERIALS AND METHODS}

\section{Isolation of a Hyperparasite From Pst-Infected Wheat Leaves}

The mycoparasite samples consisting of several wheat leaves with abnormal Pst uredinia covered by white fungal mycelia were collected during our routine experiment in a growth chamber. The wheat leaves were from susceptible cultivar Fielder inoculated with urediniospores of Pst kept at $16^{\circ} \mathrm{C}$ and $80-90 \%$ relative humidity in a growth chamber. When Pst was sporulating 14 days after inoculation, white mycelia started appearing on

TABLE 1 | Accession numbers of Lecanicillium spp. and Simplicillium spp. used in molecular characterization.

\begin{tabular}{|c|c|c|c|c|}
\hline Species & Strain number & GenBank number & Substrate (including host) & Origin/locality \\
\hline L. wallacei & CBS 101237 & EF641891/IMI 331549 & Lepidopteran larva on palm leaf & Indonesia \\
\hline L. antillanum & CBS 350.85 & NR_111097.1 & Agaric & Cuba \\
\hline L. aphanocladii & CBS 101286 & AJ292430.1 & Agaricus bisporus & United Kingdom \\
\hline L. lecanii & CBS 101247 & AJ292382/IMI 304807 & Coccidae on Coffea & West Indies \\
\hline L. lecanii & IMI 304817 & AJ292383.1 & Cordyceps confragosa & $-\mathrm{a}$ \\
\hline L. dimorphum & CBS 363.86 & NR_111101.1 & Heterodera glycines cyst & Minnesota \\
\hline L. muscarium & CBS 143.62 & AJ292388/IMI068689 & Trialeurodes vaporariorum & England \\
\hline L. muscarium & IRAN 684C & EF641892.1/EF641854 & Akanthomyces muscarius/Pleurotus ostreatus & Iran \\
\hline L. fungicola var. aleophilum & CBS 357.80 & AF324876.1 & Agaricus bitorquis & Netherlands \\
\hline L. fungicola var. aleophilum & CBS 300.70A & EF641885 & Soil from rain forest & Queensland \\
\hline L. fungicola var. aleophilum & CBS 171.80 & EF641886.1 & Agaricus bitorquis & Netherlands \\
\hline L. flavidum & CBS 112974 & EF641875 & Gomphidius glutinosus & Finland \\
\hline L. flavidum & CBS 300.70D & EF641877 & Coltricia perennis & Austria \\
\hline L. flavidum & CBS 290.80 & EF641876 & Decaying Russula nigricans & Germany \\
\hline L. fungicola var. fungicola & CBS 992.69 & NR_119653.1 & Agaricus bisporus & Netherlands \\
\hline L. fungicola var. fungicola & CBS 238.80 & EF641880.1 & Forest litter & United States \\
\hline L. fungicola var. fungicola & CBS 696.88 & EF641882 & Hypholoma capnoides & Netherlands \\
\hline S. lamellicola & CBS 116.25 & AJ292393/AF339601 & $-a$ & - \\
\hline S. obclavatum & CBS 311.74 & EF468798/AJ292394/AF339567 & Air, above sugarcane field & India \\
\hline S. obclavatum & CBS 250.76 & AY245654 & Soil & India \\
\hline S. obclavatum & CBS 510.82 & & Rust pustules on Arachis hypogea & Tamil Nadu \\
\hline S. lanosoniveum & CBS 962.72 & EF641862 & - & - \\
\hline S. lanosoniveum & CBS 704.86 & AJ292396/AF339602 & - & - \\
\hline S. lanosoniveum & CBS 962.72 & EF641862 & - & - \\
\hline
\end{tabular}

a Not available. 
Pst uredinia. The sample leaves were collected when white mycelia engulfed $50 \%$ uredinia 22 days after Pst inoculation. The leaf samples were treated with $75 \%$ alcohol for $1 \mathrm{~min}$ for surface disinfection, and the samples were washed with sterile water for five times.

The leaf samples were cut into 1-2-cm-long pieces, and the pieces were placed onto the potato dextrose agar (PDA) in Petri dishes that were incubated in darkness at $25^{\circ} \mathrm{C}$ for 5 days. Mycelial disks were cut from the edges of the fungal colony, transferred to new PDA dishes in a sterile inoculation chamber, and the new dishes were incubated under the same condition as described above for obtaining pure cultures. The purified isolates were kept on PDA slants at $4-8^{\circ} \mathrm{C}$.
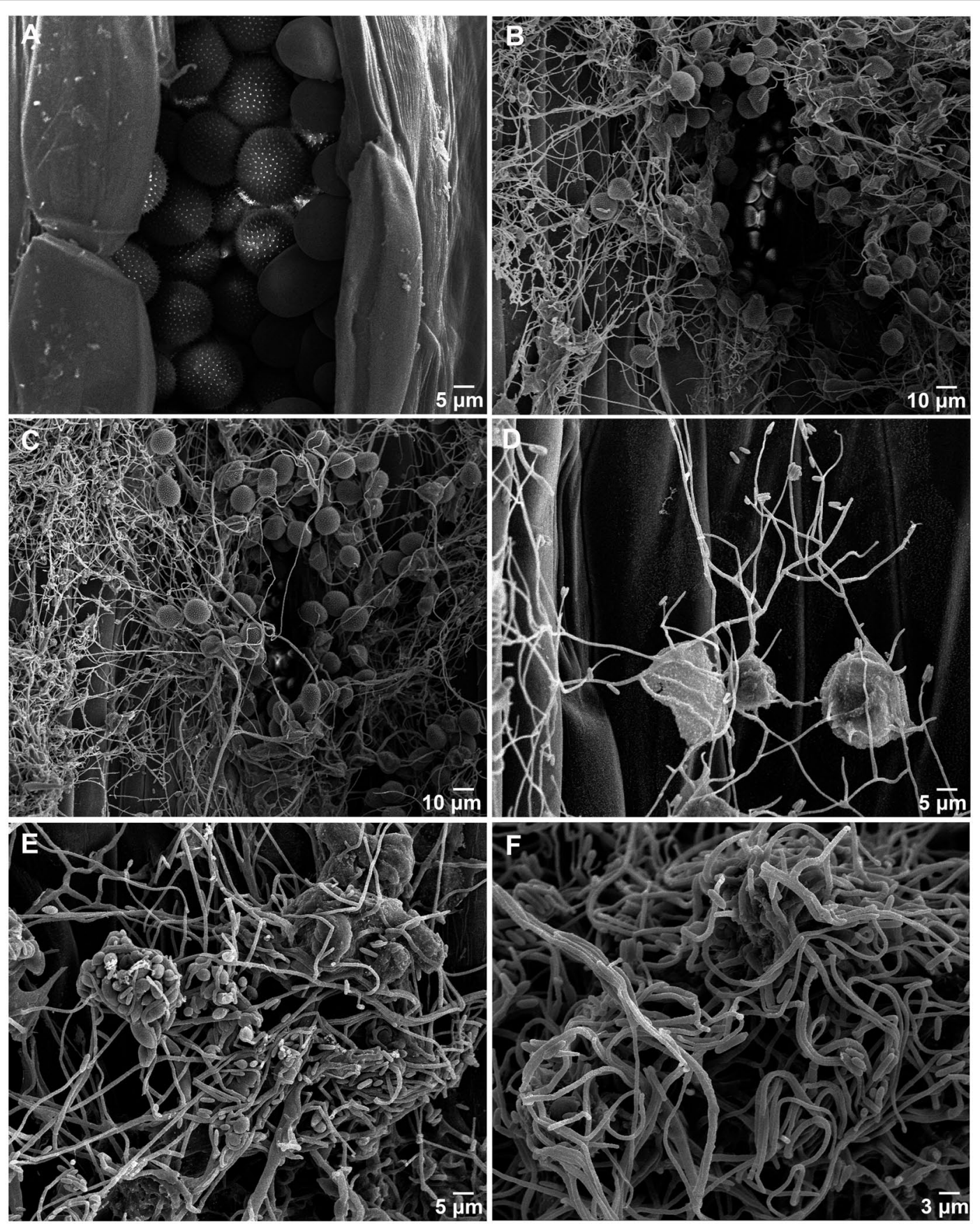

FIGURE 1 | Puccinia striiformis f. sp. tritici (Pst) uredinia and urediniospores are parasitized by Simplicillium obclavatum. (A) Uredinia before being parasitized ( $\times 900)$. (B) Uredinia were shriveled at the early infection stage of the hyperparasitic process $(\times 300)$. (C,D) The hyperparasite hyphae invading the urediniospores at the middle infection stage $(\times 800)$. $(\mathbf{E}, \mathbf{F})$ The spores of $P$ st are completely swallowed at the late hyperparasitic stage $(\times 1,500)$. 

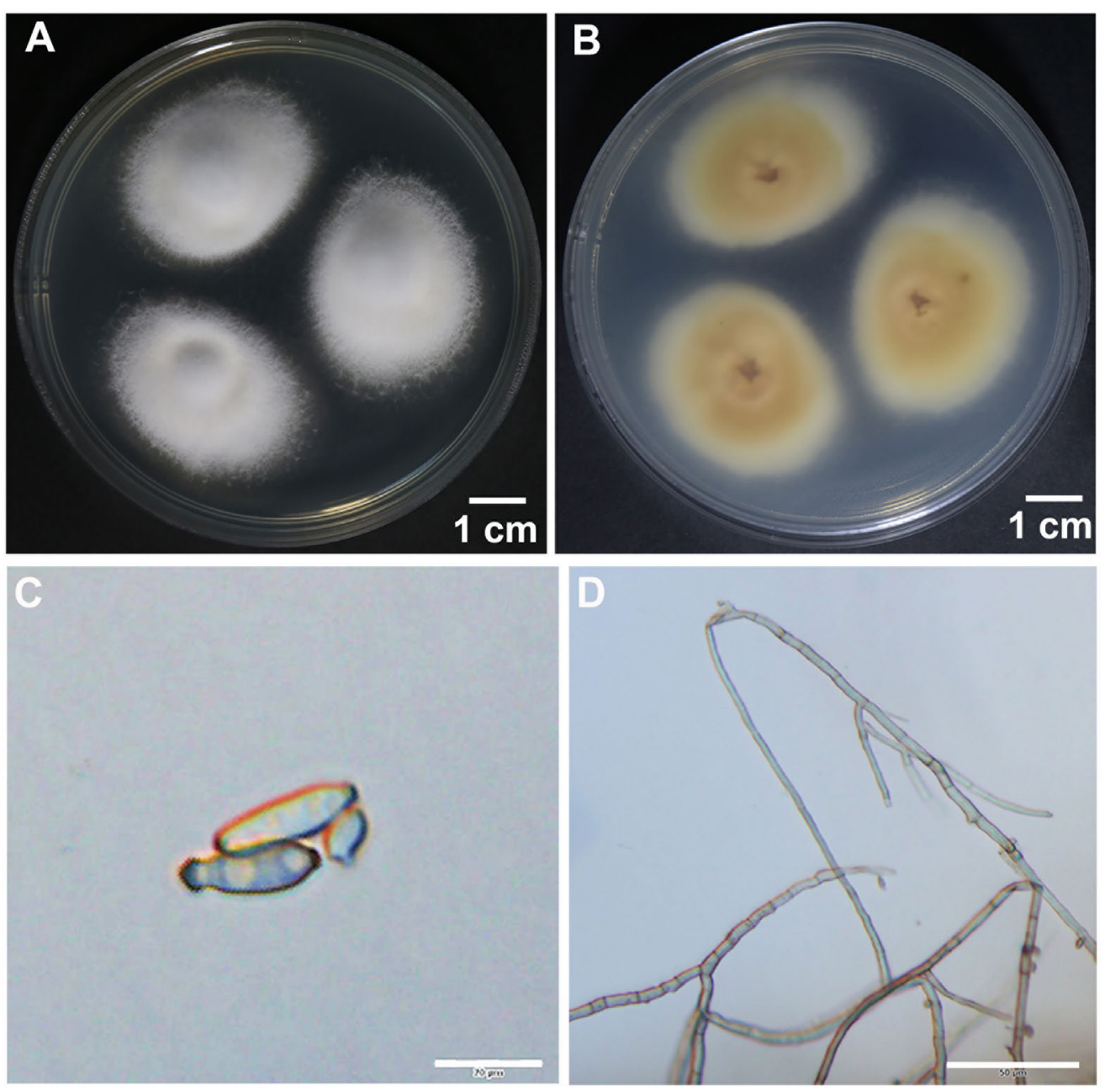

FIGURE 2 | Morphological characteristics of hyperparasite Simplicillium obclavatum. (A,B) S. obclavatum cultured on PDA medium at $25^{\circ} \mathrm{C}$ for 10 days. (A) the front side; (B) the reverse side. (C,D) Conidia and septate hypha in morphology [bar = $20 \mu \mathrm{m}$ in (C) and bar $=50 \mu \mathrm{m}$ in (D)].

\section{Observation Using a Light Microscope}

A mycelial plug ( $5 \mathrm{~mm}$ in diameter) from the edge of a growing colony was placed on to the center of a PDA plate and kept at $25^{\circ} \mathrm{C}$ for 10 days. The morphological characteristics of hyphae and conidia were observed using a light microscope (Olympus BX51T-32P01, Tokyo, Japan).

\section{Observation Using a Scanning Electron Microscopy (SEM)}

Leaf samples bearing abnormal uredinia of Pst covered by fungal mycelia were harvested and surface-washed with distilled water. The samples were cut into $5 \mathrm{~mm} \times 5 \mathrm{~mm}$ pieces and quickly placed in $4 \%$ glutaraldehyde fixative and fixed overnight at $4^{\circ} \mathrm{C}$. The leaf samples were rinsed 4 times in $0.1 \mathrm{M} \mathrm{pH} 6.8$ PBS buffer for $10 \mathrm{~min}$ each time and dehydrated with an ethanol series $(30,50,70,80$, and 90\%) for at least 15-30 min each at room temperature. The last $100 \%$ alcohol was taken three times for $30 \mathrm{~min}$ each time. The dehydrated samples were soaked in isoamyl acetate for 10-20 min and then processed with carbon dioxide drier, treated with spray-gold as previously described (Bomblies et al., 2008), and observed under a SEM (S-4800, Hitachi, Japan).

\section{Molecular Characterization}

The parasitic isolate was inoculated onto the center of a PDA plate covered with a layer of cellophane and cultured in the dark at $25^{\circ} \mathrm{C}$. Mycelia were collected five days post culture. After drying, the genomic DNA was extracted using the cetyltrimethylammonium bromide (CTAB) method (Rogers and Bendich, 1994). The internal transcribed spacer (ITS1) was used for identifying the hyperparasite. The polymerase chain reaction (PCR) solution in $20 \mu \mathrm{L}$ volume comprised $1 \mu \mathrm{L} 10 \mathrm{mM}$ ITS primer for each (ITS1-Primer: TCCGTAGGTGAACCTGCG; ITS4-Primer: TCCTCCGCTTATTGATATGC) (White et al., 1990), $2 \mu l 0.4$ mM dNTP, $10 \mu l$ PrimeSTAR ${ }^{\circledR}$ Max DNA Polymerase (Takara, China, Dalian), $2 \mu \mathrm{L}$ DNA solution, and $4 \mu \mathrm{L}$ double-distilled water. Three other representative genes, elongation factor-1 alpha (TEF1- $\alpha)$, small subunit (SSU), and 

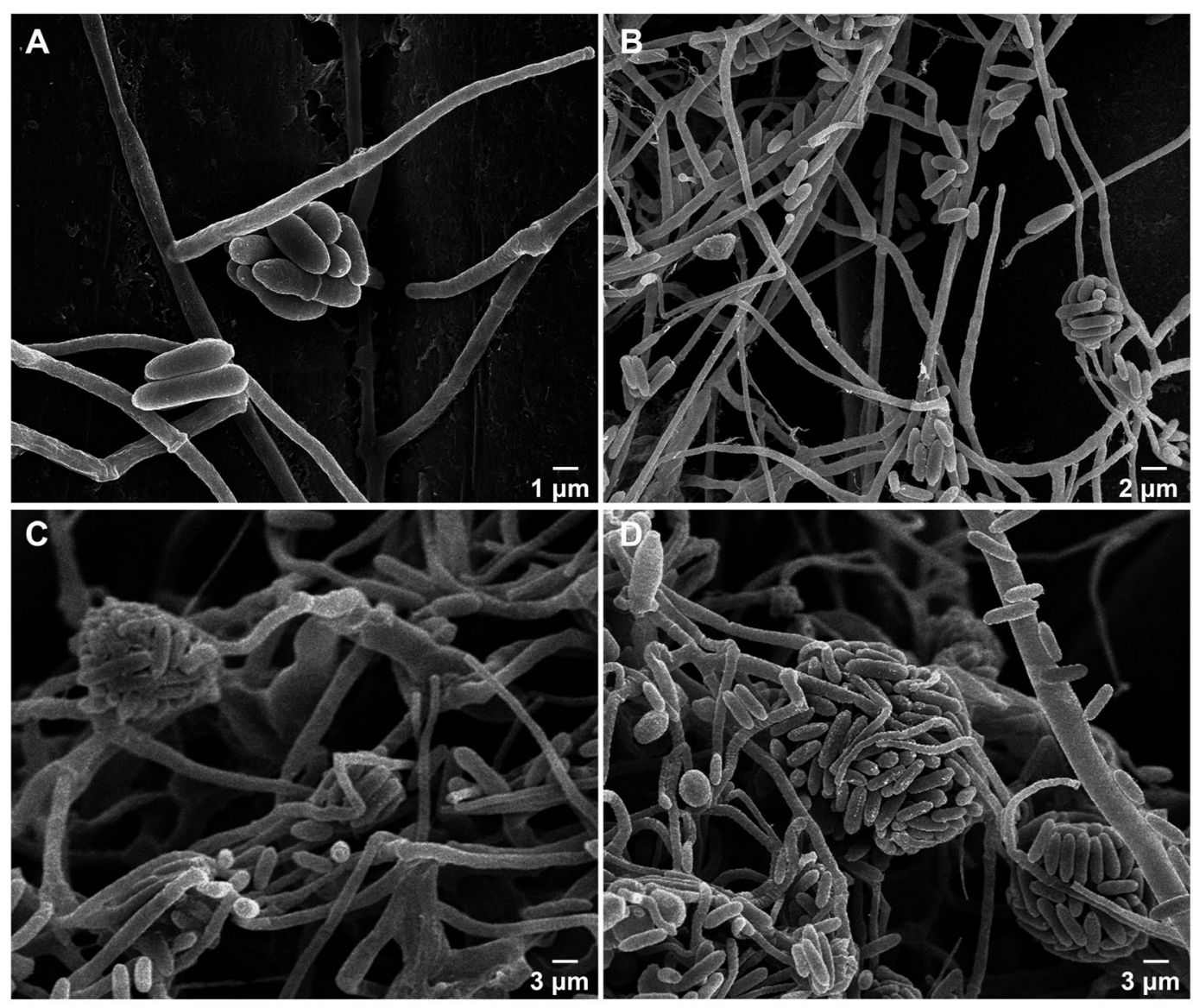

FIGURE 3 | Morphologic characteristics of Simplicillium obclavatum under a scanning electron microscope. (A-D) Mycelium, conidia and conidium ( $\times 4,000$, $\times 2,000, \times 1,500, \times 1,500$, respectively).

large subunit (LSU) of the ribosomal RNA gene, of S. obclavatum were used to confirm the ITS result. The hyperparasite DNA was amplified using specific primers of the TEF1- $\alpha$ (F: GCY CCYGGHCAYCGTGAYTTYAT; R: ATGACACCRACRGCRAC RGTYTG) (Rehner and Buckley, 2005), SSU (NS1: GTAGT CATATGCTTGTCTC; NS4: CTTCCGTCAATTCCTTTAAG (White et al., 1990), and LSU (LR0R: TACCTGGTTGATTCTGC; LR5: ATCCTGAGGGAAACTTC) (Vilgalys and Hester, 1990; Wei et al., 2019). All PCR reactions were performed as follows: denaturing at $95^{\circ} \mathrm{C}$ for $4 \mathrm{~min}, 40$ cycles (from $95^{\circ} \mathrm{C}$ for $30 \mathrm{~s}$, $60^{\circ} \mathrm{C}$ for $30 \mathrm{~s}$, to extension at $72^{\circ} \mathrm{C}$ for $40 \mathrm{~s}$ ), extension for $10 \mathrm{~min}$ at $72^{\circ} \mathrm{C}$, and keeping at $16^{\circ} \mathrm{C}$. PCR products were separated by $1.5 \%$ agarose gel electrophoresis. The PCR products were purified using the Gel Extraction Kit (CWBio, China, Beijing) and sequenced by Sangon Biotech (Shanghai, China).

\section{Phylogenetic Analysis}

The sequences of Lecanicillium spp. and Simpliciium spp. were downloaded from the NCBI website ${ }^{1}$ (Table 1) and aligned by ClustalW in software MEGA7 using the default parameters (Kumar et al., 2016). The fungal isolates were clustered based

${ }^{1}$ https://www.ncbi.nlm.nih.gov/ on their ITS sequences using the neighbor-joining (NJ) method using MEGA7, and the branch robustness was determined using 1000 bootstrap replications (Kumar et al., 2016).

\section{Hyperparasitism Assay}

The inoculations were done following the previously described methods (Zheng et al., 2017). Two-week-old seedlings of wheat (cv. Fielder) were inoculated with urediniospores of Pst isolate V26 and incubated in a dew chamber at $12^{\circ} \mathrm{C}$ in the darkness for $24 \mathrm{~h}$ and then grown in a growth chamber at $16^{\circ} \mathrm{C}$ with a 16-h light photoperiod. Twelve days after Pst inoculation, plants were inoculated with the spore suspension (concentration $1.0 \times 10^{6}$ spores $\cdot \mathrm{mL}^{-1}$ ) of the identified S. obclavatum isolate and kept in a dew chamber at $16^{\circ} \mathrm{C}$ in the darkness for $24 \mathrm{~h}$ and then returned to the growth chamber for growing under the same conditions as described above. Plants inoculated with only Pst urediniospores were used as the control. Twelve days after Pst inoculation, symptoms were observed and yellow-colored uredinia were counted using pictures analyzed with software ImageJ number counting ${ }^{2}$. Samples were collected at 12, 36, 72, 96,120 , and $168 \mathrm{~h}$ after hyperparasite inoculation (hai) and

\footnotetext{
${ }^{2}$ https://imagej.nih.gov/ij/
} 


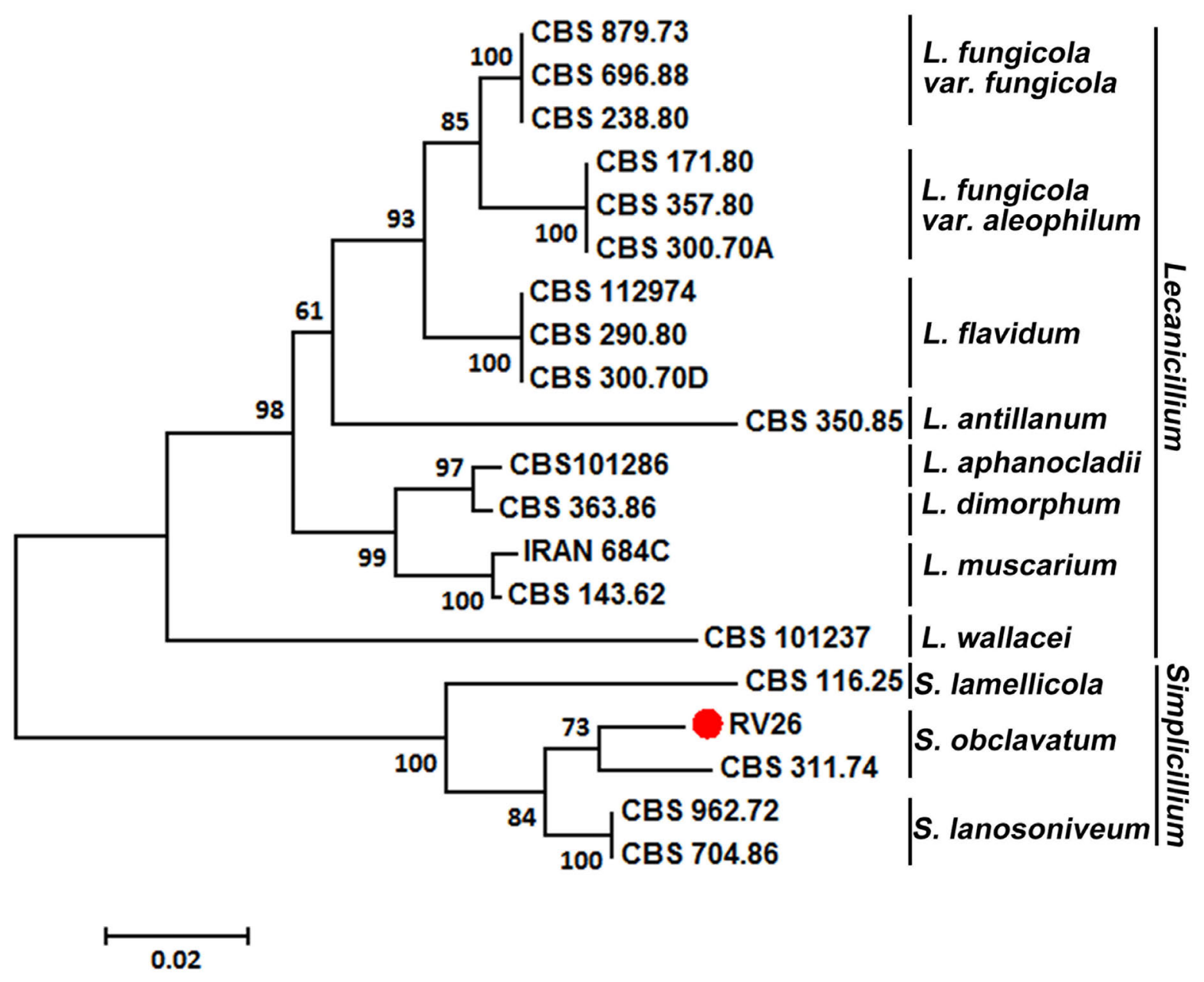

FIGURE 4 | A phylogenetic tree constructed for species of Verticillium, Lecanicillium, and Simpliciium using software MEGA7 and the neighbor-joining (HJ) method. The hyperparasitic $S$. obclavatum isolate was marked with the red dot. The percentages of replicate trees in which the associated taxa clustered together in the bootstrap test (1000 replicates) are shown next to the branches.

observed with a SEM as described above. Urediniospores were collected at 3 and 5 days after hyperparasite inoculation (dai) for evaluation of the germination rate. The experiment included three biological repeats.

\section{Pst Biomass Analysis}

Quantitative PCR (qPCR) was performed in a CFX96 Connect Real-Time PCR Detection System (Bio-Rad, Hercules, United States) to determine the Pst DNA content in the infected wheat leaves using a TB Green Premix DimerEraser (Perfect Real Time) (TaKaRa, Dalian, China). The fungal Pst-EF1 (rust elongation factor 1) and wheat TaEF1- $\alpha$ (wheat elongation factor 1 alpha) fusion plasmids were diluted into a serial concentrations $\left(10^{3}, 10^{4}, 10^{5}, 10^{6}, 10^{7}, 10^{8}\right.$, and $10^{9}$ fmolcot $L^{-1}$ ) for generation of the standard curves. Pst-EF1 primers (F: TGGTGTCATCAAGCCTGGTATGGT; R: ACTCAT GGTGCATCTCAACGGACT) and Pst-EF1 primers (F: TTCG CCGTCCGTGATATGAGACAA; R: ATGCGTATCATGGTGGT GGAGTGA) were used for qPCR analysis. Genomic DNA of the infected wheat leaves was extracted from the infected leaves at $3,5,7$, and 10 dai using the CTAB method as described above. Ct means were determined using qPCR. The Pst and wheat DNA concentrations were calculated according to the corresponding standard curves. The ratio of the Pst and wheat DNA concentrations was used to determine the Pst biomass. The experiment was conducted three times.

\section{RESULTS}

\section{Parasitization of Pst Uredinia by} S. obclavatum

The morphological changes of Pst parasitized by the $S$. obclavatum isolate on wheat leaves were observed using a SEM. Urediniospores from normal uredinia were round or oval in shape (Figure 1A). When uredinia were parasitized by the hyperparasite, the urediniospores were shriveled at the early hyperparasitic infection stage (Figures 1B-D). The hyphae 
A

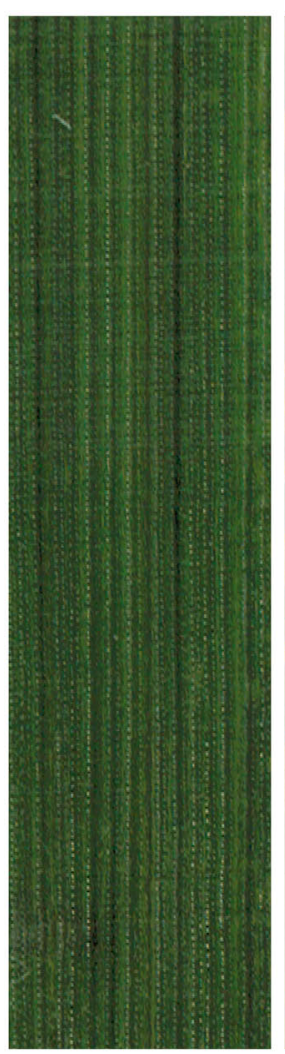

B

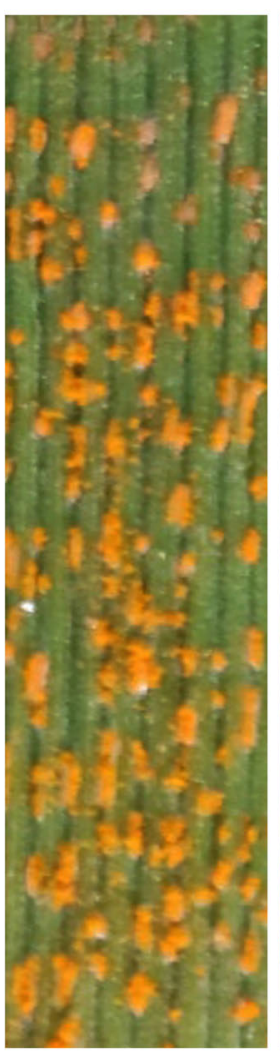

C

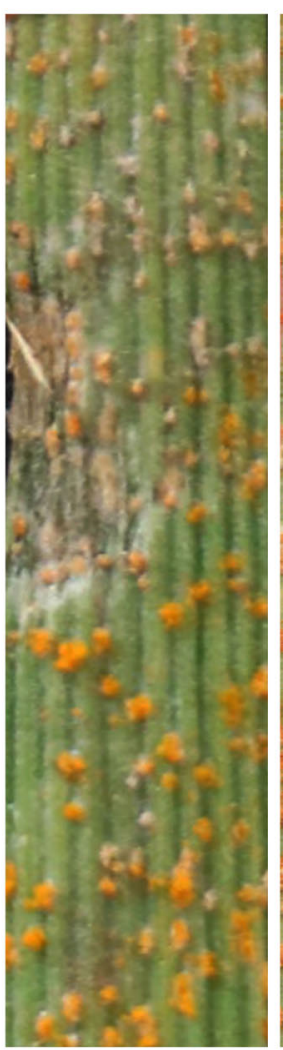

D

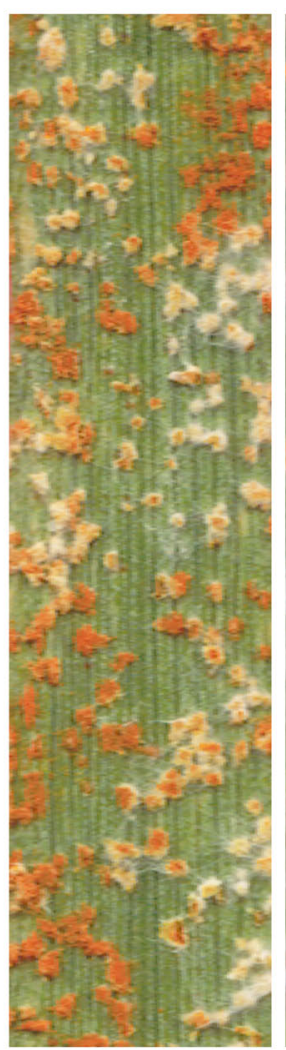

E

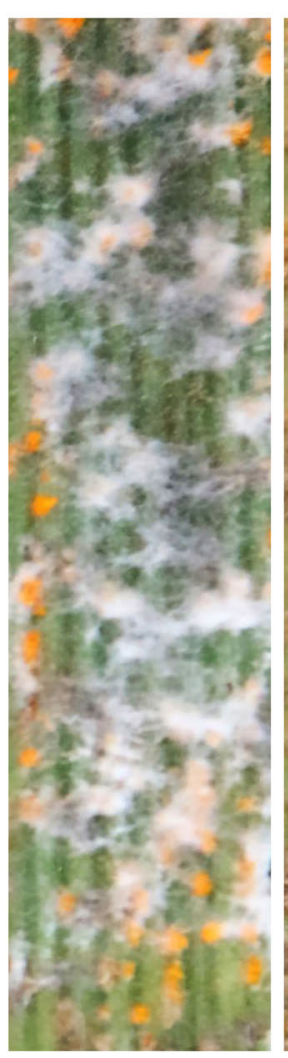

$\mathbf{F}$

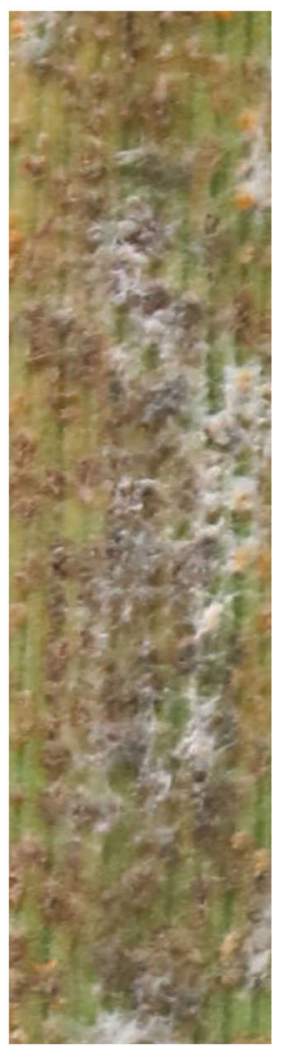

FIGURE 5 | The pathogenicity test confirming the parasitism of Simpliciium obclavatum in Puccinia striiformis f. sp. tritici (Pst) grown on wheat leaves. (A) Wheat leaves inoculated with only the spore suspension of Simpliciium obclavatum 10 days post inoculation (dpi). (B) Wheat leaves inoculated with only Pst isolate V26 12 dpi. (C-F) Wheat leaves inoculated with the spore suspension of S. obclavatum 12 days after the inoculation with Pst V26, and (C-F) show symptoms and signs at 3, 5, 7, and 10 dpi with $S$. obclavatum, respectively.

of the hyperparasitic fungus invaded Pst urediniospores at the middle infection stage (Figures 1C,D), and the urediniospores became completely swallowed at the late infection stage (Figures 1E,F). The destruction of Pst uredinial cell structures indicated that Pst uredinia parasitized by the hyperparasite lost viability.

\section{Identification of the Hyperparasite as Simplicillium obclavatum}

To identify the hyperparasite, we obtained an isolate named RV26 from hyperparasited Pst uredinia. The in vitro cultured RV26 colonies in PDA plates were white, floss in shape, and the reverse side was citron-yellow 10 days after in culture at $25^{\circ} \mathrm{C}$ (Figures 2A,B). The colonies were dense and reached $20-30 \mathrm{~mm}$ in diameter 10 days after in culture at $25^{\circ} \mathrm{C}$ (Figure 2A). Under an optical microscope, hyphae were septated, and conidia were typically obclavate to ellipsoidal, dark taupe, 20 to $35 \mu \mathrm{m} \times 2$ to $5 \mu \mathrm{m}$ in size (Figures 2C,D). These characteristics indicated that isolate RV26 is a filamentous fungus.

The ultrastructure of RV26 was further examined using a SEM. Conidia were obclavate, had 3 or more spiral phialides, and were in imbricate chains with short imbricate chains on erected conidiophores (Figure 3). The morphological features of RV26 resembled Simpliciium obclavatum (Santana et al., 2017).

The ITS1 region sequence of RV26 also supported the identification of the isolate as S. obclavatum (Figure 4). Additionally, the sequences of the RV26 fragments amplified with the TEF $1 \alpha, S S U$, and $L S U$ primers of S. obclavatum confirmed RV26 as S. obclavatum. RV26 was clustered with other four Simpliciium species and was most close to S. obclavatum isolate CBS311.74 (Figure 4). Collectively, both the morphological and molecular characteristics supported the identification of the Pst hyperparasite as S. obclavatum.

\section{The Hyperparasitism of S. obclavatum}

To confirm the ability of $S$. obclavatum isolate RV26 to parasitize Pst, we performed a hyperparasitism assay. Wheat leaves inoculated with only the spore suspension of S. obclavatum showed normal growth at $22 \mathrm{dpi}$ (Figure 5A), and those inoculated with only Pst race V26 had orange-colored normal Pst uredinia at $12 \mathrm{dpi}$ (Figure 5B). In the test of the wheat leaves inoculated with the spore suspension of $S$. obclavatum isolate RV26 12 days after inoculation with Pst isolate V26, t he hyperparasitic fungus started growing on Pst uredinia at 3 days 
A

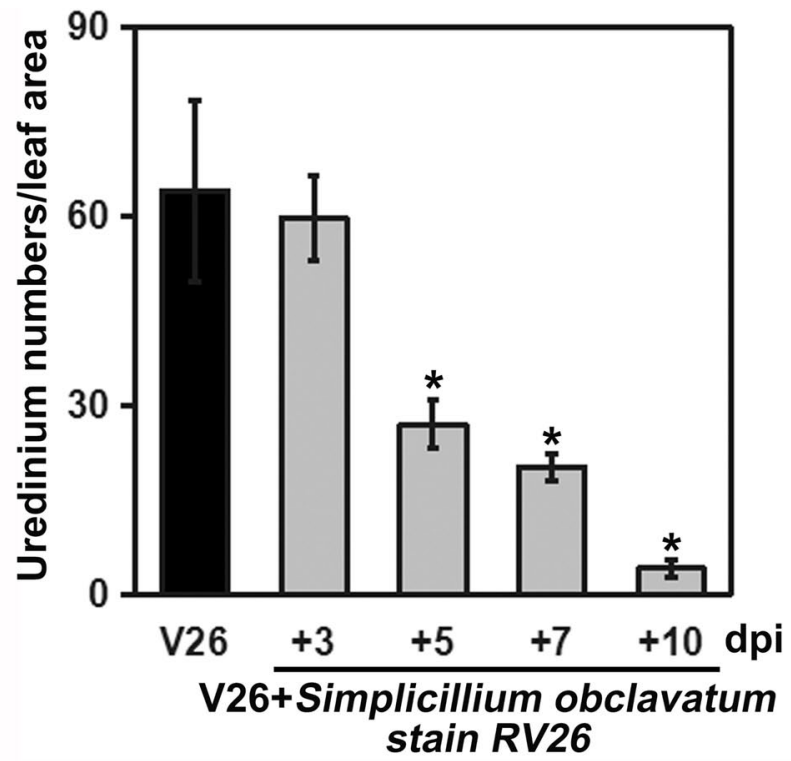

B

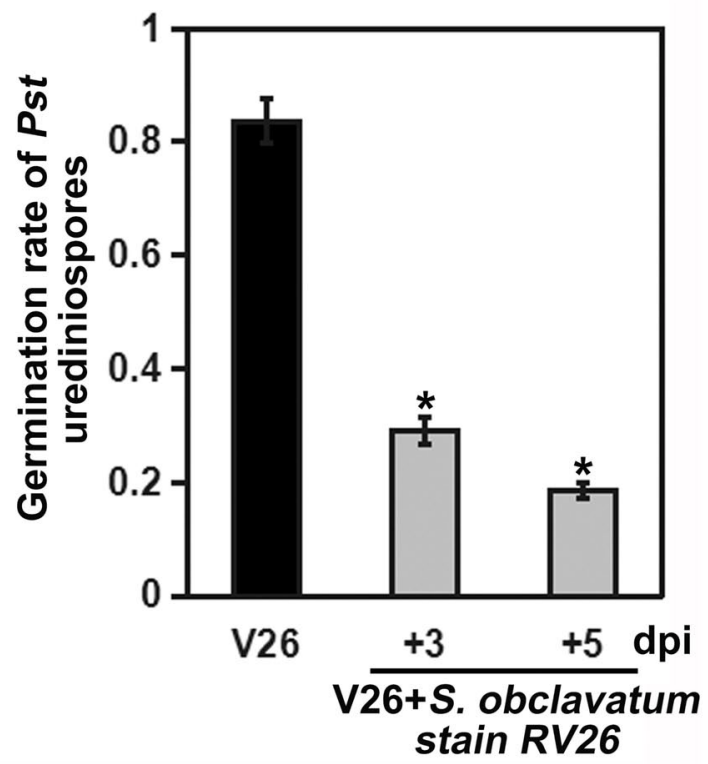

FIGURE 6 | Quantification of Puccinia striiformis f. sp. tritici (Pst) uredinia and urediniospores viability. (A) The number of uredinia counted using an ImageJ number counter. The wheat leaves were first inoculated with Pst and 12 days after inoculated (dpi) with Simpliciium obclavatum conidia. Twelve days after S. obclavatum inoculation, the number of uredinium per $\mathrm{cm}^{2}$ of wheat leaf surface was calculated. (B) Pst urediniospore germination from leaves of non-treated and treated with S. obclavatum. Pst urediniospores of non-treated with S. obclavatum were used as the control.

A

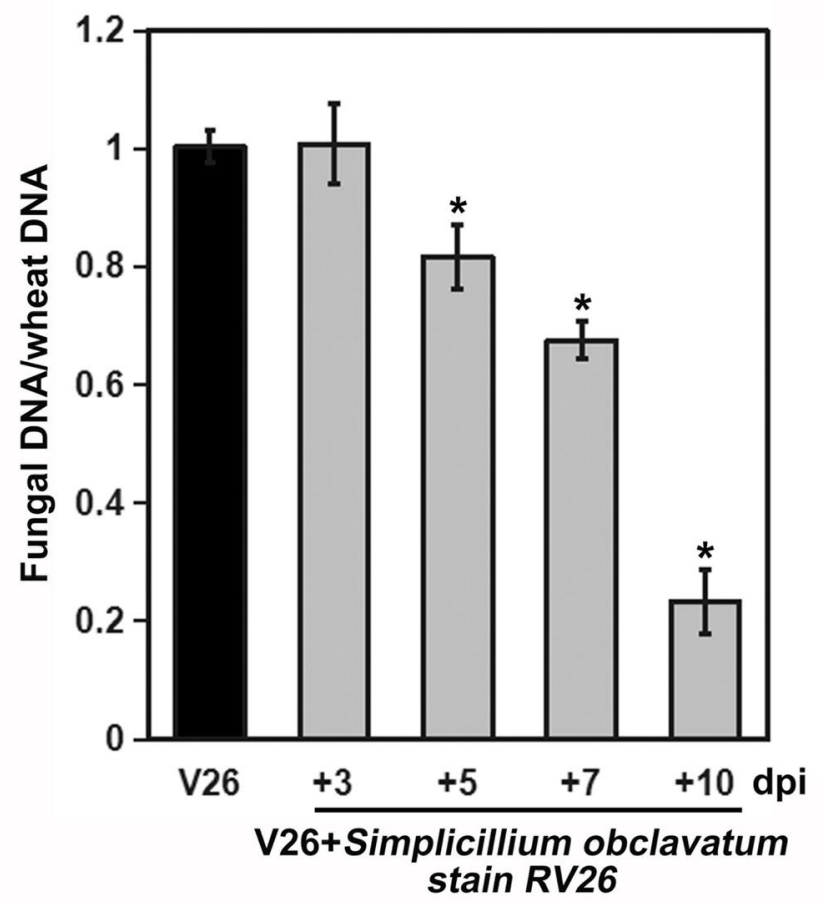

B
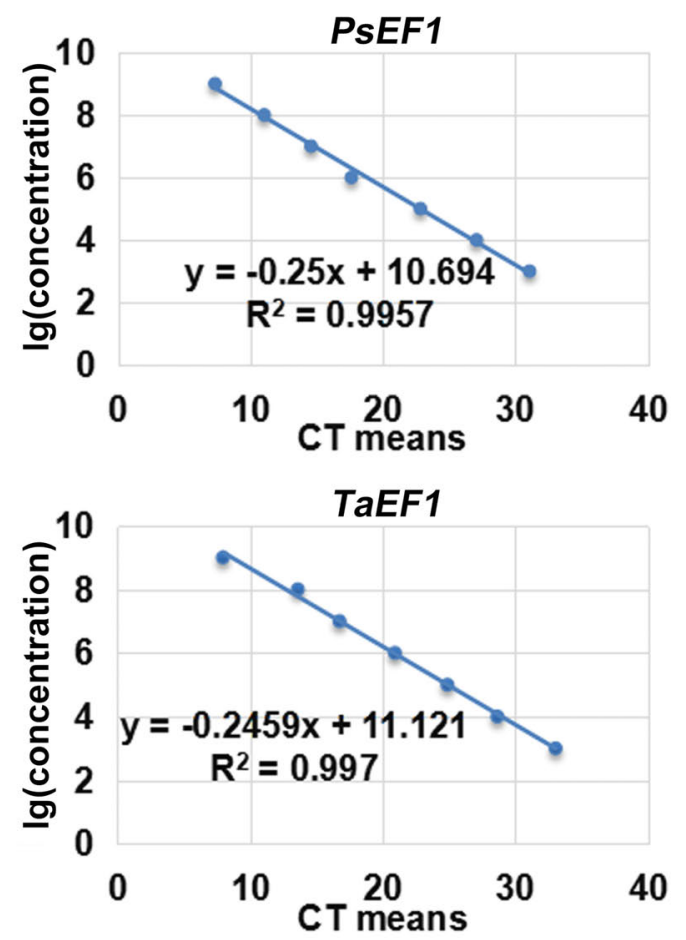

FIGURE 7 | Biomasses of Puccinia striiformis f. sp. tritici (Pst) at different times after inoculation. (A) Pst biomass was calculated based on the ratio of Pst and wheat DNA concentrations. The relative quantities of PCR product of TaEF1- $\alpha$ and PStEF1 in infected samples were calculated using the gene-specific standard curves to quantify Pst and wheat gDNA. (B) Standard curves derived from the serial dilutions. PstEF1 (Pst elongation factor 1) and TaEF1- $\alpha$ (wheat elongation factor 1 alpha). 

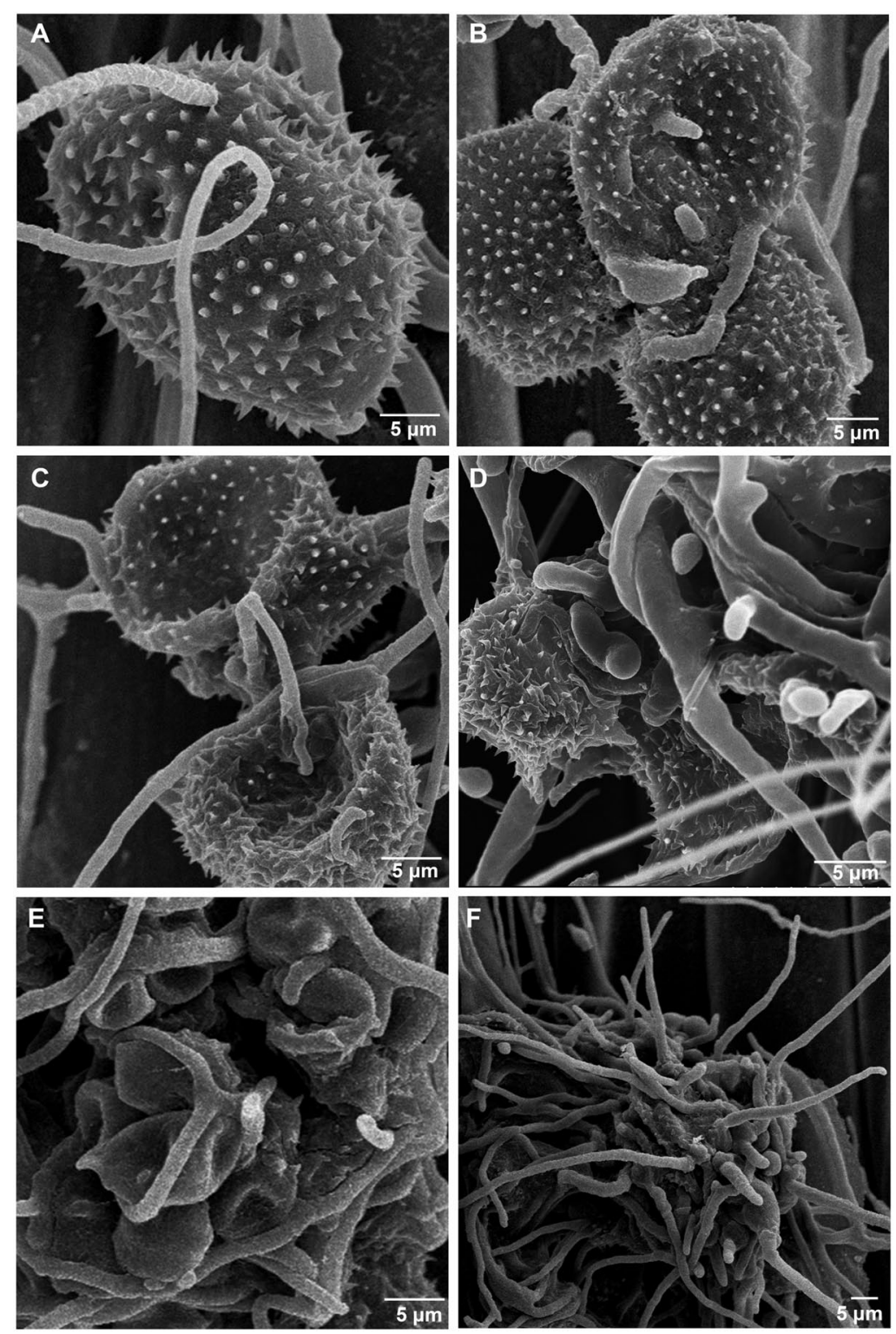

FIGURE 8 | Morphologic characteristics of Puccinia striiformis f. sp. tritici (Pst) inoculated with Simpliciium obclavatum under a scanning electronic microscope. (A) Hyphae of S. obclavatum on the surface of a Pst urediniospore ( $\times 2,000)$ at 12 hai (hours after inoculation). (B) S. obclavatum generate germ tubes at 36 hai (×1,200). (C,D) Pst urediniospores surrounded by S. obclavatum hyphae. (C) at 72 hai $(\times 1,800)$, and (D) at 96 hai $(\times 3,000)$. (E,F), S. obclavatum has colonized Pst urediniospores. $(\mathbf{E})$ at 120 hai $(\times 1,500)$ and $(\mathbf{F})$ at 168 hai $(\times 1,000)$.

after the inoculation with S. obclavatum and grew more rapidly 5 , 7, and 10 days after the hyperparasite inoculation (Figures 5C,F).

As $S$. obclavatum was growing and spreading, the number of Pst uredinia on the infected wheat leaves gradually decreased (Figure 6A). Moreover, Pst urediniospores collected from uredinia parasitized by RV26 showed a sharp loss of viability, indicated by the reduced rate of urediniospore germination (from 69\% to 83\%) (Figure 6B). It became unable to collect Pst urediniospores at 7 days after RV26 inoculation. The Pst biomass was decreased at more and more days after the inoculation (Figure 7A), which was also shown by the wheat and Pst standard curves (Figure 7B). These results indicated that $S$. obclavatum was able to parasitize Pst and effectively reduce the germination rate of Pst urediniospores.

To further confirm that the S. obclavatum isolate RV26 can effectively parasitize Pst, the morphologic characteristics of Pst inoculated with RV26 were observed using a SEM. S. obclavatum hyphae intruded into Pst urediospores through germ tubes (Figure 8A), gradually encroached Pst (Figures 8B,D), and destroyed Pst urediniospores at $5 \mathrm{~d}$ or $7 \mathrm{~d}$ after the parasite treatment (Figures 8E,F). These results confirmed that S. obclavatum RV26 were able to effectively parasitize Pst. 


\section{DISCUSSION}

It is of great significance to study mycoparasites of cereal pathogenic fungi for the exploration of biological control of cereal diseases. Identification of mycoparasites is essential to understanding the biodiversity of hyperparasites. In the present study, we discovered that S. obclavatum isolate RV26 is able to parasitize obligate bitrophic pathogen Pst. This hyperparasite is able to reduce the development of Pst urediniospores, indicating that $S$. obclavatum has the potential to be used as a biocontrol agent to control Pst.

The colony morphology and conidial size are key factors in classifying Lecanicillium spp. and Simplicillium spp. (Zare and Gams, 2008; Wei et al., 2019). However, these morphological characteristics are considerably variable in different culture conditions and environments. Therefore, it is relatively difficult to identify fungi in these genera to the species level just based on the morphological characteristics. At first, we identified the isolate RV26 parasitizing Pst as L. fungicola, as it showed very similar morphological characteristics with L. fungicola (Zare and Gams, 2001). Although the genera Simplicillium spp. and Lecanicillium spp. are very closely related, conidial morphologies and ITS sequences are different (Zare and Gams, 2001, 2008). In the present study, we used both morphological and molecular characteristics to identify the RV26 isolate as S. obclavatum.

The effects of hyperparasites on plant pathogens are the basis for biological control of plant pathogenic fungi. Extensive studies have been conducted to identify and characterize hyperparasites of plant pathogens (Kohl et al., 2009; Berendsen et al., 2012). Previous studies have reported several hyperparasitic fungi parasitic on rust pathogens, such as A. alternata, Aphanocladium album, Fusarium spp., Lecanicillium spp., and Scytalidium uredinicola (Littlefield, 1981; Moricca et al., 2001; Koç and Défago, 2008; Tsuneda et al., 2011; Zheng et al., 2017). In the present study, we found that $S$. obclavatum could invade Pst as a hyperparasitic fungus.

Some hyperparasites have been used in managing plant diseases caused by fungi (Berendsen et al., 2012; Adhikari et al., 2014; Zhong et al., 2016). The S. obclavatum isolate RV26 identified in the present study is able to reduce or stop the growth of Pst urediniospores by growing into uredinia. This parasitic ability makes RV26 a potential biological control agent

\section{REFERENCES}

Adhikari, A., Nandi, S., Dutta, S., Bhattacharya, I., and Mandal, T. (2014). Study of morphology and mycoparasitism of some antagonists of Trichoderma sp from West Bengal. J. Mount Sinai Hosp. N.Y. 1, 593-605. doi: 10.1088/0143-0807/12/ $2 / 006$

Baiswar, P., Ngachan, S. V., Rymbai, H., and Chandra, S. (2014). Simplicillium lanosoniveum, a hyperparasite on aecidium elaeagni-latifoliae in India. Aust. Plant Dis. Notes 9, 1-5. doi: 10.1007/s13314-014-0144-z

Berendsen, R. L., Kalkhove, S. I. C., Lugones, L. G., Baars, J. J. P., Wösten, H. A. B., and Bakker, P. A. H. M. (2012). Effects of fluorescent Pseudomonas spp. isolated from mushroom cultures on Lecanicillium fungicola. Biol. Control 63, 210-221. doi: 10.1016/j.biocontrol.2012.07.012 to remove Pst from infected wheat leaves. So far, it is clear that the S. obclavatum isolate is able to parasitize at the Pst sporulation stage, but it is unknown whether the fungus can infect in other Pst developmental stages. The parasitic fungus grows well under optimal humidity and temperature conditions for Pst to infect its wheat host. However, it is not clear what environmental factors affect the survival and parasitism ability of S. obclavatum. Therefore, further studies are needed to determine whether the parasitic fungus can be developed into a biological control agent for managing stripe rust under field conditions.

In summary, the present study identified $S$. obclavatum as a new hyperparasite of Pst. The fungus has the potential to be used as a biological control agent for control stripe rust. Additional research is needed to determine if the hyperparasite is environmentally friendly and, if so, to optimize conditions and develop techniques to produce and integrated the biological control agent into the current stripe rust management strategies and further to explore its potential to control other rust pathogens.

\section{DATA AVAILABILITY STATEMENT}

The raw data supporting the conclusions of this article will be made available by the authors, without undue reservation, to any qualified researcher.

\section{AUTHOR CONTRIBUTIONS}

$\mathrm{XW}, \mathrm{ZK}$, and NW conceived and designed the experiments. NW, $\mathrm{XF}, \mathrm{BL}$, and SZ conducted the experiments. NW, XF, and $\mathrm{MH}$ analyzed the data. NW, CT, and XW wrote the manuscript. XC helped revise the manuscript.

\section{FUNDING}

This study was supported by the National Key Research and Development Program of China (2018YFD0200408), the National Natural Science Foundation of China (31772150), China Agriculture Research System (CARS-3), and Shaanxi Innovation Team Project (2018TD-004).

Bettiol, W., and Várzea, V. M. P. (1992). Controle biológico da ferrugem (Hemileia vastatrix) do cafeeiro com Bacillus subtilis em condições controladas. Fitopatol. Brasil. 17, 91-95.

Bomblies, K., Shukla, V., and Graham, C. (2008). Scanning electron microscopy (SEM) of plant tissues. CSH. Protoc. 5:4933. doi: 10.1101/pdb.prot 4933

Chen, X. M. (2005). Epidemiology and control of stripe rust (Puccinia striiformis f. sp. tritici) on wheat. Can. J. Plant Pathol. 27, 314-337. doi: 10.1080/ 07060660509507230

Fisher, M. C., Henk, D. A., Briggs, C. J., Brownstein, J. S., Madoff, L. C., McCraw, S. L., et al. (2012). Emerging fungal threats to animal, plant and ecosystem health. Nature 484, 186-194. doi: 10.1038/nature 10947 
Haddad, F., Maffia, L. A., Mizubuti, E. S. G., and Teixeira, H. (2009). Biological control of coffee rust by antagonistic bacteria under field conditions in Brazil. Biol. Control 49, 110-119. doi: 10.1016/j.biocontrol.2009.02.004

Jackson, D., Skillman, J., and Vandermeer, J. (2012). Indirect biological control of the coffee leaf rust, Hemileia vastatrix, by the entomogenous fungus Lecanicillium lecanii in a complex coffee agroecosystem. Biol. Control 61, 89-97. doi: 10.1016/j.biocontrol.2012.01.004

Keener, P. D. (1954). Cladosporium aecidiicola. Thuem. and Tuberculina persicina (Ditm.) Sacc. associated with Puccinia conspicua (Arth.) mains on Helenium hoopesii A. gray in arizona. Plant Dis. Rep. 38, 690-694.

Koç, N. K., and Défago, G. (2008). Studies on the host range of the hyperparasite Aphanocladium album. J. Phytopathol. 107, 214-218. doi: 10.1111/j.1439-0434. 1983.tb00539.x

Kohl, J., Molhoek, W., Haas, B., and Geijn, H. (2009). Selection and orchard testing of antagonists suppressing conidial production by the apple scab pathogen Venturia inaequalis. Eur. J. Plant Pathol. 123, 401-414. doi: 10.1007/s10658008-9377-z

Kumar, S., Stecher, G., and Tamura, K. (2016). MEGA7: molecular evolutionary genetics analysis version 7.0 for bigger datasets. Mol. Biol. Evol. 33:1870. doi: 10.1093/molbev/msw054

Littlefield, L. J. (1981). Biology of the plant rust: an introduction. Ames, IA: Iowa state university press. Bioscience 34:1309583. doi: 10.2307/1309583

Martins, S. J., Soares, A. C., Medeiros, F. H. V., Santos, D. B. C., and Pozza, E. A. (2015). Contribution of host and environmental factors to the hyperparasitism of coffee rust under field conditions. Aus. Plant Pathol. 44, 1-6. doi: 10.1007/ s13313-015-0375-372

Moricca, S., Ragazzi, A., Mitchelson, K. R., and Assante, G. (2001). Antagonism of the two-needle pine stem rust fungi Cronartium flaccidum and Peridermium pini by Cladosporium tenuissimum in vitro and in planta. Phytopathology 91, 457-468. doi: 10.1094/Phyto.2001.91.5.457

Nischwitz, C., Newcombe, G., and Anderson, C. L. (2005). Host specialization of the mycoparasite Eudarluca caricis and its evolutionary relationship to Ampelomyces. Mycol. Res. 109, 421-428. doi: 10.1017/S0953756205002431

Rehner, S. A., and Buckley, E. (2005). A Beauveria phylogeny inferred from nuclear ITS and EF1- $\alpha$ sequences: evidence for cryptic diversification and links to Cordyceps teleomorphs. Mycologia 97, 84-98. doi: 10.2307/3762199

Rogers, S. O., and Bendich, A. J. (1994). "Extraction of total cellular DNA from plants, algae and fungi," in Plant Molecular Biology Manual, eds S. B. Gelvin and R. A. Schilperoort (Dordrecht: Springer), doi: 10.1007/978-94-011-0511-8_12

Saari, E. E., and Prescott, J. M. (1985). "World distribution in relation to economic losses," in The Cereal Rusts. Vol. II. Diseases, Distribution, Epidemiology, and Control, eds A. P. Roelfs and W. R. Bushnell (Orlando, FL: Academic Press), 259-298. doi: 10.1016/B978-0-12-148402-6.50017-1

Santana, N. J., Manuela, R. D. B., Cunha, Z. D., Eloisa, A. D. G. L., Souza, D., and Eustáquio, A. E. (2017). Evaluation of the infection process by Lecanicillium fungicola in Agaricus bisporus by scanning electron microscopy. Rev. Iberoam. Micol. 34, 36-42. doi: 10.1016/j.riam.2016.04.006

Shiomi, H. F., Silva, H. S. A., Melo, I. S., Nunes, F. V., and Bettiol, W. (2006). Bioprospecting endophytic bacteria for biological control of coffee leaf rust. Sci. Agr. 63, 32-39. doi: 10.1590/S0103-90162006000100006

Srivastava, A., Défago, G., and Kern, H. (1985). Hyperparasitism of Puccinia horiana and other microcyclic rusts. J. Phytopathol. 114, 73-78. doi: 10.1111/ j.1439-0434.1985.tb04338.x

Sun, J. Z., Liu, X. Z., McKenzie, E. H., Jeewon, R., Liu, J. K. J., Zhang, X. L., et al. (2019). Fungicolous fungi: terminology, diversity, distribution, evolution, and species checklist. Fungal Divers. 95, 227-230. doi: 10.1007/s13225-019-004 22-429
Torres, D. E., Rojas-Martínez, R. I., Zavaleta-Mejia, E., Guevara-Fefer, P., MárquezGuzmán, G. J., and Perez-Martinez, C. (2017). Cladosporium cladosporioides and Cladosporium pseudocladosporioides as potential new fungal antagonists of Puccinia horiana Henn., the causal agent of chrysanthemum white rust. PLoS One 12:e0170782. doi: 10.1371/journal.pone.0170782

Tsuneda, A., Hiratsuka, Y., and Maruyama, P. J. (2011). Hyperparasitism of Scytalidium uredinicola on western gall rust, Endocronartium harknessii. Can. J. Bot. 58, 1154-1159. doi: 10.1139/b80-143

Vandermeer, J., Perfecto, I., and Liere, H. (2009). Evidence for hyperparasitism of coffee rust (Hemileia vastatrix) by the entomogenous fungus, Lecanicillium lecanii, through a complex ecological web. Plant Pathol. 58, 636-641. doi: 10. 1111/j.1365-3059.2009.02067.x

Vilgalys, R., and Hester, M. (1990). Rapid genetic identification and mapping of enzymatically amplified ribosomal DNA from several Cryptococcus species. J. Bacteriol. 172, 4238-4246. doi: 10.1128/jb.172.8.4238-4246.1990

Wan, A. M., Chen, X. M., and He, Z. H. (2007). Wheat stripe rust in china. Aust. J. Agr. Res. 58, 605-619. doi: 10.1071/AR06142

Ward, N. A., Robertson, C. L., Chanda, A. K., and Schneider, R. W. (2012). Effects of Simplicillium lanosoniveum on Phakopsora pachyrhizi, the soybean rust pathogen, and its use as a biological control agent. Phytopathology 102, 749-760. doi: 10.1094/PHYTO-01-11-0031

Wei, D. P., Wanasinghe, D. N., Hyde, K. D., Mortimer, P. E., Xu, J., Xiao, Y. P., et al. (2019). The genus Simplicillium. Mycokeys 60, 69-92. doi: 10.3897/mycokeys. 60.38040

White, T., Bruns, T., Lee, S., and Taylor, F. (1990). Amplification and direct sequencing of fungal ribosomal RNA genes for phylogenetics. PCR Protoc. Guide Methods Appl. 18, 315-322. doi: 10.0000/PMID1793

Zare, R., and Gams, W. (2001). A revision of verticillium section prostrata. IV. The genera Lecanicillium and Simplicillium. Nova Hedwigia 73, 1-50. doi: 10.1111/ j.1756-1051.2001.tb00824.x

Zare, R., and Gams, W. (2008). A revision of the Verticillium fungicola species complex and its affinity with the genus Lecanicillium. Mycol. Res. 112, 811-824. doi: 10.1016/j.mycres.2008.01.019

Zhan, G. M., Tian, Y., Wang, F. P., Chen, X. M., Guo, J., Jiao, M., et al. (2014). A novel fungal hyperparasite of Puccinia striiformis $\mathrm{f}$. sp. tritici, the causal agent of wheat stripe rust. PLoS One 9:e111484. doi: 10.1371/journal.pone.011 1484

Zheng, L., Zhao, J., Liang, X., Zhan, G., Jiang, S., and Kang, Z. (2017). Identification of a novel Alternaria alternata strain able to hyperparasitize Puccinia striiformis f. sp. tritici, the causal agent of wheat stripe rust. Front. Microbiol. 8:71. doi: 10.3389/fmicb.2017.00071

Zhong, X., Li, S. S., Peng, Q. Y., Zhang, J. S., Kan, X. T., Zhang, G. R., et al. (2016). A polycephalomyces, hyperparasite of Ophiocordyceps sinensis, leads to shortened duration of production and reduced numbers of host ascospores. Fungal Ecol. 21, 24-31. doi: 10.1016/j.funeco.2016.03.002

Conflict of Interest: The authors declare that the research was conducted in the absence of any commercial or financial relationships that could be construed as a potential conflict of interest.

Copyright (c) 2020 Wang, Fan, Zhang, Liu, He, Chen, Tang, Kang and Wang. This is an open-access article distributed under the terms of the Creative Commons Attribution License (CC BY). The use, distribution or reproduction in other forums is permitted, provided the original author(s) and the copyright owner(s) are credited and that the original publication in this journal is cited, in accordance with accepted academic practice. No use, distribution or reproduction is permitted which does not comply with these terms. 